Meta

Journal des traducteurs

Translators' Journal

\title{
Les pionniers de l'interprétation au Canada
}

\section{Jean Delisle}

Volume 22, numéro 1, mars 1977

Histoire de la traduction au Canada

URI : https://id.erudit.org/iderudit/002529ar

DOI : https://doi.org/10.7202/002529ar

Aller au sommaire du numéro

Éditeur(s)

Les Presses de l'Université de Montréal

ISSN

0026-0452 (imprimé)

1492-1421 (numérique)

Découvrir la revue

Citer cet article

Delisle, J. (1977). Les pionniers de l'interprétation au Canada. Meta, 22(1), 5-14.

https://doi.org/10.7202/002529ar d'utilisation que vous pouvez consulter en ligne.

https://apropos.erudit.org/fr/usagers/politique-dutilisation/ 


\section{Les pionniers de l'interprétation au Canada*}

\section{L'histoire de l'interprétation au Canada s'ouvre par un kidnapping.}

Le 24 juillet 1534, après avoir élevé une croix à Gaspé, Cartier et ses hommes s'apprêtent à mettre le cap sur la France. Le capitaine malouin regrette cependant de quitter les nouvelles terres dont il vient de prendre possession officiellement sans rapporter à son roi François $\mathrm{I}^{\mathrm{er}}$ une preuve vivante de sa découverte. De son vaisseau, il voit s'approcher une barque dans laquelle il reconnaît Donnacona accompagné de trois de ses fils et de son frère ${ }^{1}$. Rendu à proximité du bâtiment, le chef iroquois se lève et laisse entendre dans une langue bizarre et à grand renfort de gestes que toute la terre qu'il est possible d'embrasser du regard lui appartient et que personne ne peut y élever de croix sans son consentement. Indifférents à la harangue passionnée de l'Iroquois, des marins sautent dans l'embarcation et contraignent les occupants à monter à bord du navire. Cartier ordonne de prodiguer tous les soins de l'hospitalité à Dom Agaya et à Taignoagny, deux des fils du grand chef. Ses ordres ne tardent pas à être exécutés à la lettre, et les deux captifs ont droit à tous les égards réservés aux souverains.

C'est ainsi qu'ont été recrutés les deux premiers interprètes du pays. Cartier inaugurait du même coup les échanges franco-québécois.

Vêtus d'une ample casaque et d'un bonnet rouge, Dom Agaya et Taignoagny débarquent en France le 5 septembre 1534 pour y apprendre le français. Cartier les destinait au métier d'interprète. Il avait compris la nécessité de disposer de truchements pour les langues du pays, si rocailleuses pour une oreille européenne, car il n'avait pas saisi un seul mot des longs discours dont on le gratifiait si généreusement dans le golfe. Lui-même apprit les rudiments de l'iroquois ${ }^{2}$, mais il ne put jamais se passer de ses interprètes.

* Cet article est tiré d'une thèse (non publiée) présentée par l'auteur en vue de l'obtention de la maîtrise en traduction à l'Université de Montréal.

1. Ces indigènes étaient des Iroquois de Stadaconé (aujourd'hui Québec) venus pêcher au large de Gaspé.

2. Cartier avait une certaine expérience des langues et avait déjà servi d'interprète. Les Malouins du xvre siècle s'engageaient volontiers comme marins sur les navires espagnols et portugais qui se rendaient aux comptoirs du Brésil. Au cours de ces voyages, il avait appris le portugais et servit à maintes reprises d'interprète aux Portugais pris en mer et gardés dans les prisons de Saint-Malo. L'explorateur termina d'ailleurs sa carrière comme courtier-interprète. Ses fonctions consistaient à traduire les pièces de bord et à renseigner les capitaines de navire sur les formalités à remplir dans les ports. 
Après un séjour de huit mois en France où ils ont acquis une connaissance ćlémentaire du français, Dom Agaya et Taignoagny repassent en Nouvelle-France. Dans les relations fort complexes qui se sont établies entre Français et Indigènes, les truchements ont joué un rôle de premier plan. Ils n'ont pas manqué de tirer parti de leur expérience européenne. Iroquois de ccur, jamais ils n'ont collaboré avec les Français lorsque les visées de ceux-ci s'opposaient aux intérêts des Stadaconéens. En tant qu'interprètes, ils se situaient à la charnière de deux civilisations : l'européenne et l'amérindienne. La fidélité pour eux a consisté à demeurer loyaux envers leur peuple. Interprètes en langue iroquoise auprès des Français, ils ont été avant tout les porte-paroje des Iroquois de Stadaconé.

À la fin de son deuxième voyage, Cartier kidnappe une seconde fois ses interprètes devenus trop intrigants, pour les expatrier sur un continent d'où ìs ne reviendront plus. En 1541, ils étaient morts. De leurs années passées en France nous savons très peu de chose, si ce n'est qu'ils vécurent en Bretagne, entrctenus en vertu d'une ordonnance de François $I^{\mathrm{er}}$ avec qui ils eurent de fréquents entretiens. Ils auraient aussi collaboré à la rédaction des deux vocabulaires francoiroquois de Jacques Cartier. La compilation de ces deux lexiques bilingues premier travail de terminologie auquel ont participé des interprètes canadiens a sans doute eu lieu en France plutôt qu'au Canada.

\section{Naissance de l'institution des interprètes-résidents}

Au tournant du Xvir siècle, la France revient en Amérique du Nord après une absence d'une soixantaine d'années et projette d'établir des postes permanents. Dès les premières tentatives de rapprochement avec les indigènes, les Français se heurtent à des barrières géographiques, culturelles, commerciales et surtout linguistiques. Devant la multiplicité de peuples et de groupes linguistiques cohabitant sur un immense territoire, le recours à des interprètes s'impose dès le début. Mais à la nécessité de surmonter l'obstacle linguistique viennent se greffer des impératifs d'ordre commercial. Aussi, pour bien saisir l'importance et le caractère original de l'institution sociale que formèrent les interprètes français en Amérique du Nord, il faut rappeler le plan mis en œuvre par Samuel de Champlain.

Par stratégie politique et commerciale, celui qu'on a surnommé le père de la Nouvelle-France, désirait établir un grand axe commercial entre Tadoussac et les Grands Lacs. Il prévoyait canaliser vers les postes du Saint-Laurent les fourrures des tribus de la coalition laurentienne ${ }^{3}$. Or, dans \& le cours normal des choses, a écrit Marcel Trudel, le trafic des fourrures aurait dû plutôt se faire de la Huronie à l'Iroquoisie et de celle-ci au fleuve Hudson. Ce qui signifie que toutes les fourrures des Grands Lacs se fussent déversées vers la Nouvelle-Amsterdam et que le comptoir du Saint-Laurent eût perdu la plus grande partie de son intérêt ${ }^{4}$. Dans les premières décennies du xvir siècle, la carte du commerce se découpait ainsi : les nations de la Confédération iroquoise alimentaient en four-

3. Cette coalition réunissait les principales tribus de la rive nord du Saint-Laurent, notamment les Hurons, les Outaouais, les Nipissingues, les Algonquins et les Montagnais; elle 4. comprenait aussi des tribus de la Confédération abénaquise.

4. Marcel Trudel, les Vaines Tentatives, Montréal, Fides, 1963, p. 366. 
rures les Hollandais et les Anglais, tandis que les nations de la coalition laurentienne traitaient leurs pelleteries avec les Français. Mais quand les ressources de l'Iroquoisie ${ }^{5}$ commencèrent à s'épuiser, les Iroquois se mirent à convoiter les fourrures du nord.

Pour acheminer les précieuses peaux de bêtes vers les comptoirs de l'est et endiguer les courants commerciaux vers le sud, Champlain et les marchands français imaginèrent de placer des interprètes dans les tribus pourvoyeuses de fourtures et confièrent à ces agents commerciaux la tâche de se rendre maître des relations avec les indigènes et de les convaincre de réserver leurs fourrures pour la traite àvec les Français. La tâche de ces interprètes-résidents n'était pas facile. Il leur fallait maîtriser l'art de l'éloquence et de la persuasion, car la Huronie située sur les bords de la baie Georgienne se trouvait à plus de six cents milles des postes du Saint-Laurent, tandis que trois cents milles seulement la séparaient des comptoirs flamands et anglais du sud. L'institution des interprètes français est née, certes, de la nécessité de vaincre l'obstacle des langues, mais elle a été inspirée surtout par des impératifs commerciaux et une stratégie politique savammeat orchestrée.

Le programme de Champlain ouvrait une carrière nouvelle à une élite de jeunes, prêts à affronter la rude vie des bois. Pour veiller à leurs intérêts politiques et commerciaux, les Français avaient senti le besoin de disposer d'ambassadeurs dignes de confiance ${ }^{\circ}$. Outre le monopole des communications, les interprètes détenaient celui des relations politiques et commerciales avec les peuples de la coalition; ils ont formé une classe d'hommes qui occupent une place de premier plan dans la vie du Canada primitif. Polyglottes, agents commerciaux, diplomates, guides, explorateurs, telles furent les principales fonctions assumées par ces pionniers de l'interprétation au pays.

\section{Étienne Bralé}

Étienne Brûlé fut une des figures les plus hautes en couleur de la colonie naissante. Il arrive au pays l'année de la fondation de Québec et très tôt il ressent un vif attrait pour la vie sauvage et aventureuse. Son désir rejoignait le plan que Champlain mûrissait, et celui-ci l'envoya séjourner chez les Algonquins durant l'hiver de 1610-1611. C'est avec une vive émotion qu'il l'accueillit au printemps * habillé à la sauvage, qui se loua du traitement de ceux-ci ». Et il ajoute : "[II] avait bien apris leur langue ?. "Nous sommes le 13 juin 1611 et un personnage nouveau apparaît en Nouvelle-France : l'interprète-résident.

Connu surtout comme truchement de la langue huronne, Brâlé servit d'intermédiaire à Champlain à l'occasion des cérémonies d'alliance ou d'expéditions de guerre. Il aurait aussi participé à la rédaction du dictionnaire de la langue huronne

5. Territoire situé au sud du Saint-Laurent et du lac Ontario et s'étendant approximativement du Richelieu à l'emplacement actuel de Rochester.

6. L'expérience d'interprètes indigènes n'ayant pas été heureuse, on change donc de méthode : des interpretes français vivront d'abord dans une tribu et se mettront à l'ecole des indigènes. Marcel Trudel, op. cit., p. 150.

7. Samuel de Champlain, CEuvres (éd. Biggar), t. II, p. 368. 
du Frère Gabriel Sagard. Plus que tout autre interprète, Brûlé fut profondément * transformé » par ses contacts de tous les jours avec les Indieis. Adoptant intégralement leur mode de vie, il en vint à abandonner la civilisation européenne et à renier ses origines. En s'identifiant aux indigènes, les interprètes qui, à l'instar de Brûlé, s'indianisaient, ont largement contribué à atténuer le choc culturel produit par la rencontre de l'homme de la Renaissance et de l'homme de l'âge néolithique. Ils établissaient un pont entre deux groupes qui n'étaient pas vraiment " contemporains \$.

À vivre chez les indigènes, Brûlé finit par agir et penser comme eux, au point de devenir, affirme Sagard, "d'aussi légère croyance qu'eux ${ }^{8}$ ». Il respectait à la lettre les superstitions et les convictions des indigènes et, comme ses amis, il devint fétichiste. II s'était si bien assimilé qu'un jour il confia au religieux Sagard que, s'étant trouvé en danger de mort, il n'avait su réciter, pour toute prière, que le bénédicité ${ }^{\circ}$.

Établi à demeure chez les Hurons, Brûlé ne négligea pas d'organiser la traite à chaque printemps et ne faillit jamais à ses engagements envers les marchands qui lui versaient une généreuse rémunération pour son travail. Il est mort en 1633, assassiné par les membres de la tribu de l'Ours. Son exécution fut suivie d'un festin anthropophagique et ses ossements furent enterrés dans des bois près du village. Sa mort funeste privait Champlain d'un ambassadeur incomparable auprès des Hurons, et les marchanđs, d'un agent commercial très habile. Malgré ses excentricités, son libertinage et son paganisme, il a rendu d'innombrables services à la colonie et à certains missionnaires. Pour leur part, les Hurons perdaient un frère d'adoption. Ce n'est en fait que pour des \& raisons d'État» qu'ils se résignèrent à le faire mourir. Ils lui étaient si attachés qu'ils ne parvinrent jamais à effacer de leur mémoire le triste souvenir de son assassinat. Ne réussissant pas à retrouver la tranquillité d'esprit, ils incendièrent leur village en 1634. Cinq ans après la mort du truchement, les remords les assaillaient encore.

Ces faits témoignent de la fascination qu'exerça Brûlé sur l'esprit des indigènes. Sa forte personnalité les avait littéralement subjugués. L'existence nomade qu'il mena pendant plus de vingt ans était devenue pour lui une seconde nature. Il ne reprit jamais goût à la vie civilisée. Les documents de l'époque nous ont laissé l'inage d'un homme robuste, indépendant et audacieux, doué d'un grand talent pour les langues et animé d'un incomparable esprit d'initiative. Il faut saluer en cet homme un grand interprète du Canada.

\section{Jean Nicolet}

Originaire de Cherbourg, Jean Nicolet peut être considéré comme l'interprète le plus représentatif du régime français. Il alliait un caractère heureux, un grand dynamisme et une excellente mémoire ${ }^{10}$. Chez les Népissingues où il a vécu pendant quinze ans, il logeait dans sa propre cabane. C'est dans ce « petit chez-

8. Gabriel Sagard, Histoire du Canada, Paris, Ed. Tross, 1866, t. III, p. 754.

9. Ibid., t. II, p. 430 .

10. Barthélémy Vimont, Relations des Jésuites (éd. Thwaites), t. XXIII, p. 274. 
soi $\gg$ qu'il rédigea ses Mémoires, malheureusement perdus aujourd'hui. La vie nomade l'obligeait souvent à * passer plusieurs fois les sept \& huict jours sans rien manger, [et] il fut sept semaines entières sans autre nourriture qu'un peu d'écorce de bois ${ }^{11}$ ". Ces dures conditions de vie n'empêchèrent pas Nicolet d'assimiler la langue des Algonquins et celle des Hurons pendant ses deux premières années de séjour en milieu indigène. Il ne tarda pas non plus à mériter toute la confiance de ses frères d'adoption. Il sut d'ailleurs si bien gagner leur amitié et leur admiration qu'il fut nommé au Grand Conseil de la redoutable nation des Népissingues et mérita le surnom d'Achirra, c'est-à-dire « homme double ».

Jean Nicolet mourut prématurément par noyade en 1642 à la hauteur de Sillery. Pendant les vingt-quatre ans qu'il pratiqua le métier d'interprète en Nouvelle-France, Nicolet exerça toujours un grand ascendant sur les Indiens. C'est à ce signe qu'on reconnaissait un bon interprète. En plus d'être passé maître des langues algonquine et huronne, il apprit à saisir les subtilités de l'esprit des peuples qu'il fréquenta. Sans cette connaissance intime des indigènes, il n'aurait pu maintenir la paix entre les nations alliées des Français, ni diriger le flot des pelleteries vers le cœur du pays. Il s'acquitta en outre fort bien des missions diplomatiques et des ambassades de pacification qu'on lui confia. Durant l'occupation de Québec, il se réfugia chez les Hurons et s'attacha à faire obstacle à toutes les tentatives des Anglais pour amener les Indiens à commercer avec eux. Prenier Français à voguer sur le lac Michigan et à se rendre au Mississippi, Nicolet fut un explorateur aussi remarquable qu'Étienne Brûlé et un interprète non moins éminent.

\section{Les autres interprètes de la première heure}

Outre Étienne Brûlé et Jean Nicolet, les Normands Nicolas Marsolet, Jean et Thomas Godefroy, Jacques Hertel et François Marguerie ont été des figures bien connues et pittoresques des premiers temps de la colonie ${ }^{12}$. Il en est de même du Parisien Jean-Paul Godefroy, qui fut matelot avant d'être interprète, d'Olivier Letardif et de Nicolas Vignau. Les textes de l'époque font aussi mention de Jean Manet et de Jean Richer, interprètes chez les Népissingues, et d'un truchement d'origine grecque qui sert les Français au pays en 1627 et 1628 .

L'institution des interprètes-résidents n'a pas été abolie à la mort de Champlain survenue en 1635. Régulièrement des jeunes gens s'enfonçaient dans les bois pour faire l'apprentissage du métier de truchement. Qui ne connaît Pierre Boucher, Charles Le Moyne et Guillaume Couture, trois éminents interprètes qui ont acquis une réputation enviable à plus d'un titre dans la colonie. Guillaume Couture, à qui l'on prête un caractère peu commode, connut une vie fort mouvementée. La première fois qu'il se rendit en Iroquoisie, cinq guerriers l'assaillirent et le traînèrent dans leur village pour le torturer. Ils lui arrachèrent les ongles, lui brisèrent les jointures, lui percèrent lentement la paume des mains et lui scièrent un doigt avec un coquillage. On lui fit faire ensuite la « tournée » des villages agniers; il recevait

11. Barthélémy Vimont, op. cit., p. 276.

12. Marsolet et Marguerie sont originaires de Rouen, Nicolet de Cherbourg, les frères Godefroy, de Lintot, et Hertel, de Fécamp. La Normandie a fourni de nombreux interprètes à la colonie à cette époque. 
la bastonnade à chaque nouvelle bourgade. Ce traitement était de nature à lui faire abandonner à tout jamais la vie aventureuse des bois et le périlleux métier d'interprète. Mais ayant été épargné et donné à la famille du chef qu'il avait tué au moment de sa capture, Couture continua de se perfectionner dans la langue iroquoise et de déceler les intentions secrètes des chefs. Il s'adapta rapidement à son nouveau mode de vie et acquit même l'estime et la considération de ceux qui avaient voulu le mettre à mort. Il fut le premier Français à acquérir une grande influence en Iroquoisie. Ironie du sort, l'interprète revint à Trois-Rivières en 1645 en compagnie d'émissaires agniers. Vêtu à l'indienne, il eut de la peine à se faire reconnaître des Français. Agissant avec l'autorité d'un capitaine, il avait su convaincre les Iroquois de signer un traité de paix.

\section{La vie en tribu}

Dès leur arrivée dans une tribu, les interprètes adoptaient intégralement le mode de vie de leurs hôtes et ne tardaient pas à participer aux festins, danses et rites de sorcellerie qui se mêlaient aux actes de la vie quotidienne des Indiens. Ceux qui vivaient chez les semi-sédentaires comme les Hurons ou les Iroquois, jouissaient d'un meilleur sort et menaient une existence moins pénible que leurs collègues qui séjournaient chez les nomades, tels les Algonquins ou les Montagnais. Vivre dans une tribu nomade constituait une des expériences les plus pénibles qui soient pour un Européen. Excellente école pour faire l'apprentissage d'une langue, la vie en milieu indigène comportait de nombreux dếsagréments. Comme leurs hôtes, les jeunes Français vivaient dans des wigwams ou des maisons communes - quand ils ne logeaient pas tout simplement à l'enseigne des étoiles. Quant à la nourriture, les Européens la trouvaient souvent infecte et mal apprêtée. Malgré tout, les interprètes n'hésitaient pas à modeler leur comportement sur celui de leurs hôtes ${ }^{13}$. Certains d'entre eux furent choisis pour garder vivante la mémoire d'un défunt, ce qui était un honneur. Ceux-là partageaient encore plus intimement les privations, les travaux et les misères de leur famille d'adoption. Dans un désir de s'assimiler totalement aux Indiens, beaucoup d'interprètes se vêtirent comme eux. Comme épreuves d'initiation, ils devaient rivaliser d'habileté avec les indigènes et sortir vainqueurs de divers défis et concours d'aptitude physique. Il leur fallait entrer en compétition à la course, en canot ou à la raquette. Pour les Amérindiens, le courage, la force physique et l'adresse comptaient parmi les plus grandes vertus, et les victoires des jeunes interprètes forçaient leur admiration. Vivre chez les indigènes comportait aussi de grands risques. Presque tous les interprètes furent faits prisonniers à un moment ou l'autre de leur carrière et connurent les tourments de la torture, certains y laissant même leur vie.

Ces quelques faits relatifs à la vie des interprètes chez les Amérindiens témoignent de la double préoccupation qui animait ces hommes : s'intégrer totalement au milieu indigène et acquérir de l'ascendant sur les tribus. L'intégration totale et volontaire, tout en favorisant une meilleure compréhension de la mentalité et des

13. Gabriel Sagard note : « Les Français mestnes mieux instruits \& élevés dans l'Ecole de la Foy deviennent Sauvages pour si peu qu'ils vivent avec les Sauvages. $\$ O p$. cit., t. I, p. 166. 
langues indigènes, raffermissait les liens et la sympathie des tribus de la coalition envers les Français.

\section{Les interprètes judiciaires et militaires}

Jusqu'à la fin du régime français, et même après la Conquête, marchands, administrateurs, militaires et fonctionnaires de la justice eurent recours aux services d'interprètes. "Les marchands, écrivit Bacqueville de La Potherie, auraient pour 100000 escus de marchandises qu'ils ne pourraient vendre une livre de tabac sans le secours de leurs interprètes ${ }^{14}$.

À Montréal, les tribunaux faisaient fréquemment appel à des interprètes, comme c'est le cas de nos jours encore. Il y avait dans cette ville des interprètes pour toutes les langues indiennes et d'autres pour l'anglais et le hollandais, langues des marchands des colonies du sud. Au nombre de ces interprètes, on peut citer les noms de Jean Quenet, Pierre Couc dit LaFleur, René Cuillerier dit Léveillé, André David, Jean Legras, Françoise Goupil (une des rares femmes avec Élisabeth Courc à avoir exercé le métier d'interprète sous le régime français), Robert Poitiers du Buisson, Louis-Hector Piot de Langloiserie et Louis Daveluy dit Larose. Bien entendu, tous ces interprètes n'exerçaient pas la profession à plein temps. Leur statut correspondait à celui des «pigistes » actuels. Jean Quénet était maître chapelier de métier, René Cuillerier et André David, colons, et Robert du Buisson, cornmis au contrôle de la marine.

Il existait aussi sous le régime français une autre catégorie d'interprètes : les interprètes militaires. Ces hommes faisaient partie des troupes régulières et occupaient souvent un poste de commandement dans un fort éloigné. Parmi les plus célebres, mentionnons Paul Le Moyne de Maricourt, Joseph Godefroy de Vieuxpont, François Hertel, Jean-Paul Legardeur. Enfin, l'armée du marquis de Montcalm comptait en 1757 plus de 1700 Indiens de tribus différentes et dix interprètes qui traduisaient les discours à tour de rôle. Ces interprètes informaient aussi le marquis des doléances et exigences des indigènes.

\section{Les langues indiennes}

La carte linguistique de la Nouvelle-France se morcelait en de nombreux dialectes dérivés de deux familles linguistiques principales : algonquienne ou algique et huronne-iroquoise. Même si toutes les langues dérivaient de l'une ou l'autre de ces deux familles, il fallait néanmoins \& qu'il y ait un Truchement particulier pour les Montagnais, un autre pour les Sauvages de l'île (les Algonquins) \& un pour les Népissingues » bref, qu'il y ait « partout des Truchements divers, pour n'ignorer rien des langues, \& d'une infinité de mots qu'ils ont de différens les uns des autres ${ }^{15} \gg$. Chaque langue constituait une barrière linguistique en soi. Les tentatives de francisation des Indiens aboutirent à des échecs. Maîtres de la scène économique, les pourvoyeurs de fourrures ne se donnaient pas la peine d'apprendre

14. Bacqueville de La Potherie, cité par Pierre Margry, Découvertes et établissements des Français dans l'ouest et dans le sud de l'Amérique septentrionale, t. V, p. 186.

15. Gabriel Sagard, op. cit., t. II, p. 335. 
la langue d'un client qu'ils tenaient à leur merci. D'ailleurs, ils se sont habitués très tôt aux interprètes, les seuls qui, à l'exception des missionnaires, franchissaient la barrière linguistique.

Pour nous renseigner sur les caractéristiques des langues indigènes, leurs difficultés et les peines qui accompagnaient leur étude, il ne faut pas compter sur les Indiens, qui ne connaissaient pas l'écriture fondée sur un alphabet, ni sur les interprètes, indifférents à l'étude systématique des langues. Hommes essentiellement pratiques, certains d'entre eux répugnaient même à communiquer leur connaissance des dialectes. Il faut donc nous tourner vers les missionnaires et scruter leurs écrits pour connaître plus intimement les idiomes locaux. Les religieux furent les premiers à organiser méthodiquement l'étude des langues pour en faciliter l'apprentissage, assurer la continuité de leur travail et mener à bien leur cuvre d'évangélisation. On a même dit que leur première démarche en Nouvelle-France fut une démarche linguistique. Qu'avaient donc de si particulier ces langues pour que le Père Paul Le Jeune écrive à leur sujet : "Peuplez votre mémoire de tous les mots qui signifient chaque chose en particulier, apprenez le nœud ou la syntaxe qui les allie, vous n'êtes encore qu'un ignorant. Vous pourrez bien avec cela vous faire entendre des Sauvages, quoi que non pas toujours, mais vous ne les entendrez $\operatorname{pas}^{18}>$ ?

À la grande diversité de dialectes s'ajoutait l'instabilité du vocabulaire. L'absence de grammaire, de règles fixes et d'une tradition d'écriture rendait les langues «changeantes \& sujettes à la caprice des hommes ${ }^{17} \gg$; elles évoluaient rapidement et d'une manière désordonnée. La prononciation, surtout celle des sons gutturaux, constituait une autre pierre d'achoppement pour les Français. Le Père Le Jeune avoue humblement qu'il prononce les langues indiennes comme un Allemand prononce le français ${ }^{18}$. Il suffisait de manquer une seule syllabe d'un mot pour en changer la signification. Celui qui n'était pas rompu à cette gymnastique vocale tronquait involontairement les mots, ce qui avait pour effet de soulever des éclats de rire parmi les indigènes. Les intonations, les aspirations, la cadence constituaient autant de pièges pouvant entraîner des contresens ou des incompréhensions. Un accent placé sur la mauvaise syllabe changeait la signification d'un mot. «En népissingue, rapporte Sagard, kidauskinne, avec une certaine façon de prononcer veut dire, tu n'as point d'esprit, \& par un autre ton signifie : tu as menty ${ }^{19}$. On devine les conséquences fâcheuses que pouvait produire une mauvaise intonation.

La traduction du vocabulaire abstrait du français posait un problème encore plus épineux que l'instabilité des langues, la prononciation ou l'audition. Interprètes et missionnaires se virent dans la nécessité d'inventer toute une terminologie correspondant à des notions abstraites ou spirituelles. Il fallait « mille gesticulations et chimagrées ${ }^{20} \gg$ pour faire comprendre tout concept qui ne tombait pas sous les sens. Si les langues aborigènes manquaient de mots abstraits, elles

16. Paul Le Jeune, Relation de 1634-1635, t. VII, p. 26.

17. Gabriel Sagard, op. cit., t. 1I, p. 333.

18. Paul Le Jeune, op. cit., p. 92

19. Gabriel Sagard, op. cit., p. 142.

20. Pierse Biard, Relation de 1612-1614, t. II, p. 16. 
foisonnaient, en revanche, en termes descriptifs traduisant par le détail l'univers quotidien et les gestes familiers. Et cette surabondance constituait un autre obstacle. Traduire littéralement «Le vent pousse la neige » par routin rakhineou couné faisait rire les indigènes puisqu'eux n'employaient que le seul mot piouan ${ }^{21}$.

En sonime, contrairement aux missionnaires qui s'astreignirent à l'étude des langues amérindienies pour les raisons évoquées précédemment, les interprètes ne s'appliquaient pas à organiser systématiquement l'étude des idiomes pour surmonter l'obstacle de la communication. C'est ce que le récollet Le Clercq veut dire quand il écrit que les «truchements n'avoient aucune connaissance des langues, qu'ils ne sçavoient que par routine ${ }^{22} »$. Il importait assez peu, en fait, que les interprètes rendent scrupuleusement toutes les nuances des discours. Leur rôle consistait plutôt à s'emparer de l'esprit des tribus afin de mener une propagande efficace auprès d'elles. Cette tâche exigeait qu'ils sachent rivaliser d'éloquence et trouver des images, des métaphores et des allégories conformes au génie des Amérindiens. On attendait d'eux qu'ils puissent transposer un message dans une forme de pensée tout à fait différente de l'esprit français.

On ne saurait trop insister sur l'importance pour l'interprète d'exceller dans l'art oratoire, et Nicolas Perrot, un des rares interprètes à nous avoir laissé des écrits ${ }^{23}$, nous a transmis de beaux exemples de son habileté à convaincre. Il est reconnu que les Indiens étaient de grands orateurs doués d'une vive imagination et d'un sens aigu de l'image, des métaphores et des allégories. Les Français s'émerveillaient devant la clarté, la simplicité et la vivacité des exposés des indigènes, qui savaient faire appel à l'intelligence comme à l'imagination de leurs auditeurs. Logique, pathétisme et style imagé, tels étaient les principaux éléments de leur rhétorique. O:, de par leurs fonctions, les interprètes devaient rivaliser d'éloquence avec eux et modeler leurs discours sur cette rhétorique, la seule capable d'émouvoir les indigènes. C'est par leur talent d'orateur qu'ils parvenaient à s'imposer, ce qui s'explique par le fait que les chefs indiens eux-mêmes étaient choisis principalement pour leur don oratoire et leur pouvoir de persuasion. $\mathrm{Si}$ Nicolas Perrot a été pendant près de quarante ans le principal artisan des relations franco-indigènes dans l'Ouest, c'est surtout grâce à ses talents d'orateur.

\section{Conclusion}

La Barre, successeur de Frontenac, écrivit en 1682 « [qu'] une des personnes qui est la plus nécessaire au service du Roi en ce pays est un interprète ${ }^{24}{ }$. En effet, le rôle des interprètes sous le régime français ne se limitait pas à celui de * truchement \$ pour les langues. Ces mandataires des marchands et des autorités civiles auprès des tribus (les interprètes officiels, comme Nicolas Perrot, ajoutaient sous leur signature la mention * Interprète du Roy \$) remplissaient la double

21. Paul Le Jeune, op. cit., p. 28.

22. Chrestien Le Clercq, P̈remier Etablissement de la foy dans la Nouvelle-France, $\mathrm{t}$. I, p. 328.

23. Nicolas Perrot, Mémoire sur les maurs, coustumes et relligion des sauvages de l'Amérique septentrionale (réimpression de l'édition du R.P. J. Tailhan), Montréal, éd. Elysée, 1973.

24. Ia Barre, cité par Hervé Biron, Dictionnaire biographique du Canada, t. II, p. 236. 
fonction de commis-voyageurs et d'ambassadeurs. Ils vécurent dans l'ombre des gouverneurs et des personnages éminents de nos annales. Leur profonde connaissance du milieu indigène en faisait des conseillers tout désignés pour les affaires indiennes.

Mais plus important encore fut leur rôle dans la rencontre de deux civilisations. Par leur commerce journalier avec les indigènes, les interprètes ont contribué largement à atténuer le choc des cultures. Chargés d'exercer une influence directe sur les autochtones, les interprètes se trouvaient dans la nécessité d'établir des liens psychologiques avec l'homme du néolithique américain. Il leur fallait cultiver une profonde intelligence de la mentalité de l'indigène pour s'èmpreindre de sa vision du monde. Apprendre une langue, n'est-ce pas fondamentalement apprendre le langage d'une société, c'est-à-dire ses signes culturels, économiques et sociologiques. C'est à ce niveau que s'établissait la vraie communication, la véritable interprétation. L'interprète qui avait acquis le plus d'ascendant sur les Indiens n'était pas nécessairement celui qui possédait le mieux les langues amérindiennes - bien que beaucoup fussent de remarquables polyglottes - mais celui qui comprenait intimement l'âme indienne. Ayant su capter la confiance de l'Amérindien en établissant une communion d'esprit avec lui, les interprètes ont formé une sorte de "groupe tampon » entre deux civilisations mises brutalement en contact. La France choisit, pour des raisons économiques évidentes, de mener une politique d'alliances qui lui permettait d'étendre son influence à l'intérieur du continent américain et d'assimiler indirectement l'autochtone. L'interprète a été l'homme clé de ces visées.

Cet intermédiaire a bien joué son rôle parce qu'il ne s'est pas contenté de traduire les langues indiennes, mais également la manière de penser, le mode de vie et la civilisation de l'Indien. Un des interprètes de cette période n'a-t-il pas mérité le surnom d' " homme double » et un autre, celui d' " homme deux fois »? C'est dire à quel point ces interprètes avaient su s'accorder à la mentalité de l'Amérindien. Cet idéal ne demeure-t-il pas vrai au $\mathrm{xx}^{\mathrm{e}}$ siècle?

JEAN DELISLe 